\title{
Sandwich Structure in Al-Cu(-Au) Alloys-Characterization by Atomic-Resolution HAADF-STEM and EDXS-STEM
}

Yunhe Zheng ${ }^{*}$, Yixian Liu ${ }^{1}$, Nick Wilson ${ }^{2}$, Shiqi Liu ${ }^{1}$, Xiaojun Zhao ${ }^{3}$, Houwen $\mathrm{Chen}^{3}$, Jinfeng $\mathrm{Li}^{4}$, Ziqiao Zheng ${ }^{4}$, Laure Bourgeois ${ }^{1,5}$ and Jian-Feng $\mathrm{Nie}^{1}$

1. Department of Materials Science and Engineering, Monash University, Victoria, Australia.

2. CSIRO Manufacturing, Victoria, Australia.

3. College of Materials Science and Engineering, Chongqing University, Chongqing, PR China.

4. School of Materials Science and Engineering, Central South University, Changsha, PR China.

5. Monash Centre for Electron Microscopy, Monash University, Victoria, Australia.

* Corresponding author: yunhe.zheng@monash.edu

The Al-Cu system has been a classical example for studying phase transformations in alloys, and it is also the base for developing Al alloys with high-strength [1]. In this system, the precipitation process usually involves the formation of GP zones, $\theta^{\prime \prime}, \theta^{\prime}$, and $\theta$, and the microstructure is thought to be wellunderstood $[1,2]$. In our investigation of the distribution of precipitates in an $\mathrm{Al}-4 \mathrm{Cu}(\mathrm{wt} \%)$ alloy using high-angle annular dark-field (HAADF) scanning transmission electron microscopy (STEM), we found, surprisingly and interestingly, a GP zone/Al/ $\theta^{\prime}$ sandwich structure in which one or multiple GP zone(s) form adjacent to the broad surface of $\theta^{\prime}$ (Figure 1). In such structure, the number of sandwiched $\{002\}_{\alpha}$ planes between the broad surface of $\theta^{\prime}$ and the GP zone adjacent to it, and that between every two neighboring GP zones, is always three. Density functional theory calculations indicate that this number of sandwiched $\{002\}_{\alpha}$ planes corresponds to an energy minimum. Although the crystal structure of the individual precipitate phases in this alloy system has been well established, to authors' knowledge, such GP zone/Al/ $\theta^{\prime}$ sandwich structure has never been reported. While trace alloying additions are effective in enhancing the age hardening response of Al-Cu alloys [3], we show that, with the example of Al-4Cu$0.1 \mathrm{Au}(\mathrm{wt} \%)$ alloy, such sandwich structure can also form in the presence of alloying additions. In the ternary alloy, it is hard to distinguish $\mathrm{Au}$ from $\mathrm{Cu}$ as they both have higher atomic number than $\mathrm{Al}$. Although energy-dispersive X-ray spectroscopy (EDXS) is suitable for identifying different elements, for Al alloys, mapping at atomic-scale remains a challenging problem due to electron beam damage. In this work, from the sandwich structure, we have successfully acquired atomic-resolution EDXS maps on an FEI Titan $\mathrm{G}^{2}$ 60-300 ChemiSTEM, and the results show that Au atoms distribute mainly in the central part of $\theta^{\prime}$ but not in GP zones (Figure 2). Density functional theory calculations confirm our experimental observations, and further clarify that it is energetically favorable for Au to substitute for the $\mathrm{Cu}$ sites, instead of $\mathrm{Al}$ sites, in the central part of $\theta^{\prime}$. Our findings are unique as they suggest a new way of alloy design — strengthening by tailoring the shape of precipitates [4].

References:

[1] DA Porter and KE Easterling, in "Phase transformations in Metals and Alloys", (Chapman and Hall, London) p. 291.

[2] JF Nie in "Physical Metallurgy", ed. DE Laughlin, K Hono, (Elsevier, Oxford) p. 2009.

[3] Y Chen et al., Acta Mater. 125 (2017), p. 340.

[4] The authors gratefully acknowledge funding from the Australian Research Council. 

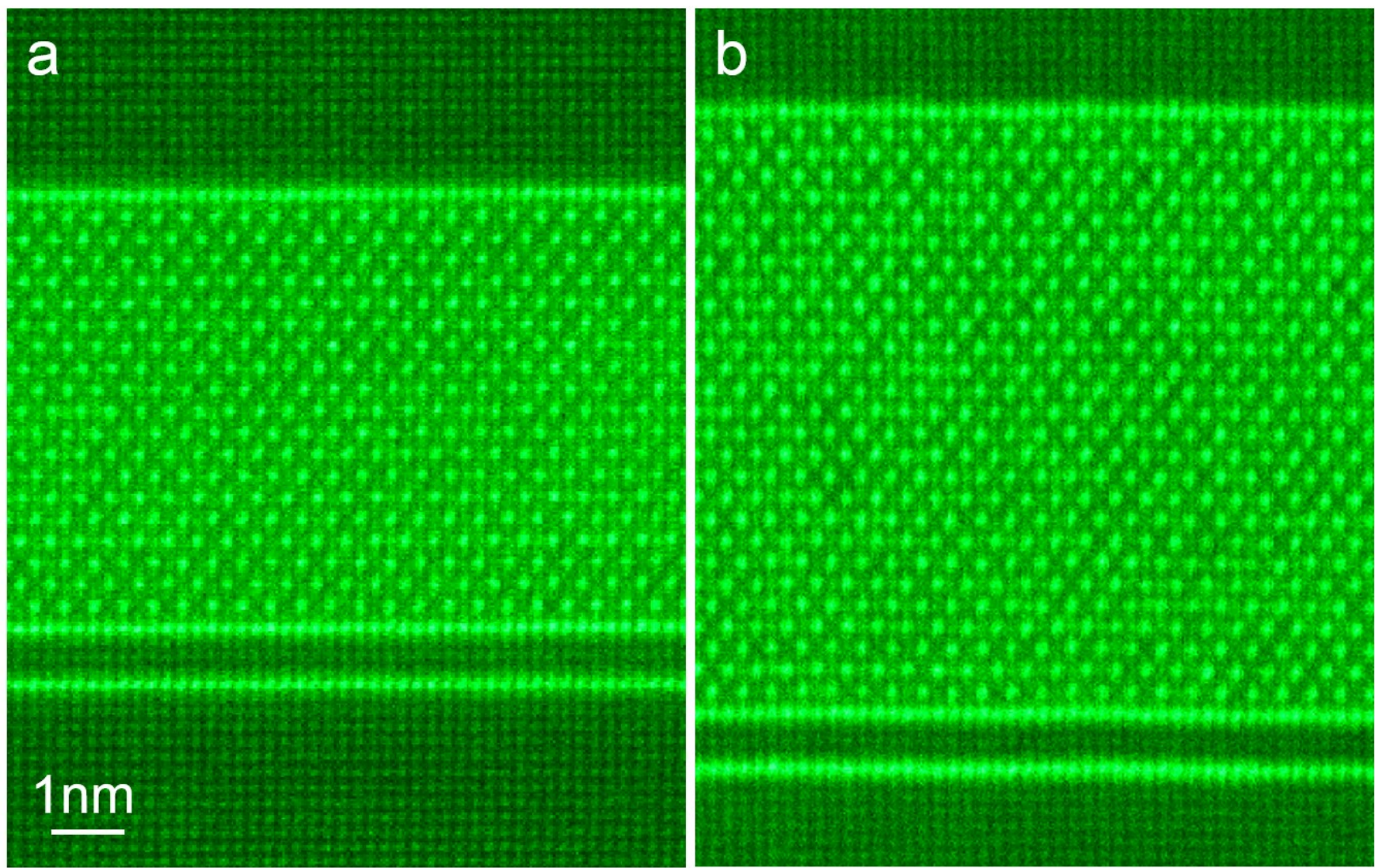

Figure 1. $<100>_{\alpha}$ HAADF-STEM images of GP zone/Al/ $\theta^{\prime}$ sandwich structures in the Al-4Cu (wt $\left.\%\right)$ alloy. The $\theta^{\prime}$ precipitate plate is of (a) 10 and (b) 14 unit-cell thickness.

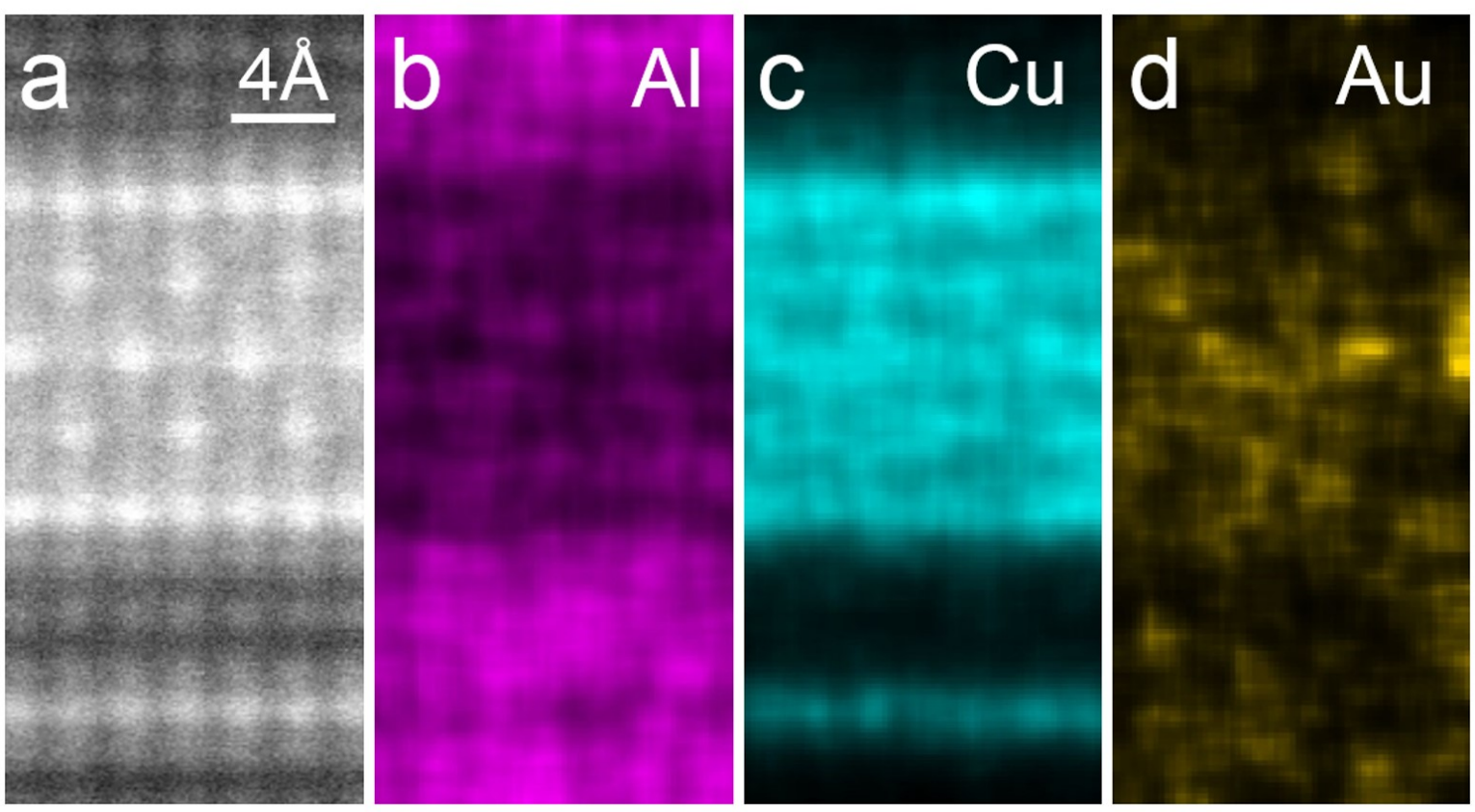

Figure 2. (a) $<100>_{\alpha}$ HAADF-STEM image and (b-d) corresponding atomic-resolution EDXS-STEM maps of a sandwich structure in the $\mathrm{Al}-4 \mathrm{Cu}-0.1 \mathrm{Au}(\mathrm{wt} \%)$ alloy. 